\title{
Gene expression analysis using long-term preserved formalin- fixed and paraffin-embedded tissue of non-small cell lung cancer
}

\author{
THERESE A.S. JACOBSON ${ }^{1}$, JOACHIM LUNDAHL ${ }^{2}$, HÅKAN MELLSTEDT ${ }^{1}$ and ALI MOSHFEGH ${ }^{1}$ \\ ${ }^{1}$ Department of Oncology and Pathology, Karolinska Institutet; ${ }^{2}$ Department of Clinical Immunology \\ and Transfusion Medicine, Karolinska University Hospital, 17176 Stockholm, Sweden
}

Received November 17, 2010; Accepted January 10, 2011

DOI: 10.3892/ijo.2011.936

\begin{abstract}
This study was performed to evaluate RNA extraction and gene expression analysis of non-small cell lung cancer (NSCLC) using formalin-fixed and paraffin-embedded (FFPE) specimens stored for more than 20 years by quantitative PCR (qPCR) and DNA microarrays. Long-term preserved FFPE materials enable large retrospective studies correlating molecular features with therapeutic response and clinical outcome. qPCR was used to evaluate RNA extraction methods and to compare DNA microarray gene expression profiles of FFPE and fresh frozen (FF) tissue. The Ambion RecoverAll kit appeared to be suited for RNA extraction of long-term preserved FFPE tissues. Microarray analysis using the Affymetrix platform displayed a high degree of correlation for endogenous control genes comparing FF and FFPE tissues and identified known NSCLC signature genes in both specimens. We conclude that high quality gene expression signatures can be recognized using the Affymetrix gene expression platform on FFPE tissue stored for more than 20 years. However, a general interpretation must be done with caution as different FFPE procedures have varying effects on RNA quality.
\end{abstract}

\section{Introduction}

Archives of FFPE tissue specimen represent a unique source for cancer research. Using FFPE material, large retrospective studies correlating molecular features with therapeutic response and clinical outcome, can be performed (1). In recent years, microarray technology has become an important tool for research, especially in the field of cancer. The ability to simultaneously analyze the expression of thousands of genes become important to correlate gene expression patterns with numerous

Correspondence to: Professor Håkan Mellstedt, Department of Oncology, Cancer Centre Karolinska, Karolinska University Hospital Solna, SE-171 76 Stockholm, Sweden

E-mail: hakan.mellstedt@karolinska.se

Key words: RNA, formalin-fixed and paraffin-embedded, fresh frozen, qPCR, gene expression clinical parameters leading to better prediction of tumor behaviour in the individual patient (2). The main reason why FFPE derived RNA is not routinely used in gene expression studies is that the process of formalin fixation and paraffin embedding affects the RNA quality. The process is designed to preserve tissue morphology, but modifies RNA and degraded RNA molecules with limited Poly-A tail and cross linking artefacts are commonly noted (3-7).

Recent studies that evaluate the use of FFPE material for RNA extraction and qPCR present varying results. The majority of studies include fresh tissues or cells which are formalinfixed and paraffin-embedded at the time of the study, in order to mimic a fixation process (1,3-4,7-12). However, FFPE tissues in histopathology departments have in general been stored for several years and since storage time may affect downstream applications, it seems more relevant to use stored tissues to evaluate the methods for nucleic acid extraction and gene expression analysis.

Several studies use only a few control genes $(1,3,7,8,12)$ and the selection of genes for qPCR has been discussed (12). Microarray analysis is the technique of choice to analyze a broader spectrum of genes, but we found only a few studies evaluating the performance of FFPE RNA in microarray analysis. Moreover, to our knowledge, no study has aimed to evaluate the use of FFPE tissues stored for over 20 years for RNA extraction and qPCR followed by microarray.

Taken together, no consensus exists concerning RNA extraction methods, reliable qPCR and microarray analysis of long time stored FFPE tissues or which endogenous control genes to be used. Therefore, the present study was designed to evaluate the use of long time stored FFPE specimens of NSCLC in RNA extraction and gene expression using qPCR and microarray as compared with fresh frozen (FF) specimens. Three FFPE RNA extraction methods were compared in terms of RNA quality and endogenous control gene expression using qPCR. In parallel, RNA was extracted from FF specimens from the same patient. Microarray analysis was done using both FFPE and FF RNA. The results were compared to qPCR of endogenous control genes in both specimens to evaluate the correlation between FF and FFPE methods as well as the correlation between qPCR and microarray. Moreover, the expression of identified NSCLC signatures were compared to published data validating the consistency of the gene expression analysis. 


\section{Materials and methods}

Tissue materials. The present study was approved by the Local Ethics Committee. One FF NSCLC tissue and one stored FFPE NSCLC block originating from the same patient were collected at the Department of Pathology, Karolinska University Hospital, Stockholm, Sweden. The FFPE NSCLC block had been stored for more than 20 years and was embedded using standard protocols. A solid and dense section of the tissue was used for embedding. Melted paraffin was poured into a steel mould. Warmed forceps was used to place the tissue in paraffin. A cassette was placed upon the mould and more paraffin was added. The paraffin was stiffened at $4-8^{\circ} \mathrm{C}$.

Study design. Three RNA extraction methods from Roche (Basel, Switzerland), Ambion (Austin, TX, USA) and Qiagen (Hilden, Germany), designed for FFPE material was used (Fig. 1). The methods were compared using qPCR. For the qPCR analysis, two different concentrations of input cDNA were used (20 and $100 \mathrm{ng}$ ) and 32 human endogenous control genes defined by Applied Biosystems (Applied Biosystems, Foster City, CA, USA) were examined. Based on the results of qPCR analysis, RNA from the Ambion FFPE kit was further analyzed with microarray. Amplification of RNA prior to microarray analysis was performed using Nugen Technologies (San Carlos, CA, USA). Nugen has developed amplification kits both for FFPE and FF materials: WT- ovation FFPE RNA amplification System V2 (Nugen FFPE), Ovation FF RNA Amplification System V2 (Nugen V2 FF) and Ovation FF Pico RNA Amplification System (Nugen PICO FF). The Nugen FFPE kit, designed for FFPE material was only used for FFPE tissue. The Nugen Pico FF kit, designed to target small amounts of FF RNA ( $>500 \mathrm{pg})$ and the Nugen V2 FF kit designed to target total FF RNA, were only used for FF tissue. Affymetrix standard amplification protocol (Affy FF) designed for FF RNA was also included as the standard method for amplification.

RNA extraction. The RNA extraction methods were all performed according to the manufacturer's recommendations. All extractions were made in duplicates. The RNA extractions using FFPE tissue were of sections taken from the same FFPE block. The RNA extractions using FF tissue were from the same tissue sample. Quality control of RNA was performed using the Nanodrop Technologies ND 1000 (Wilmington, DE, USA). Absorbance (OD-value) ratio (260/280) higher than 1.8, but not exceeding 2.1 were considered to be of high purity.

qPCR analysis. The three FFPE RNA extraction kits (Roche, Ambion and Qiagen) were compared by examining the expression of human endogenous control genes using qPCR. RNA (50 ng) extracted from each of the three kits was converted to cDNA using the High Capacity RNA to cDNA kit (Applied Biosystems, Foster City, CA, USA) according to manufacturer's recommendations. The thermal cycler \#1294, Techne Progene Peltier (Scientific Support Inc., Hayward, CA, USA) was used for the reverse transcription reaction. cDNA of two different concentrations (20 and $100 \mathrm{ng}$ ) was added to TaqMan Express Human Endogenous Control Plate (Applied Biosystems, Foster City, CA, USA) prepared with 32 human endogenous control assays. qPCR was performed using Applied Biosystems 7500 real-time PCR system.

\section{Amplification of RNA from $F F$ and FFPE tissues}

Nugen Technologies, Inc WT- Ovation FFPE RNA Amplification System V2 (Nugen FFPE). RNA (100 ng) extracted from FFPE NSCLC material using the Ambion RecoverAll Total Nucleic Acid Isolation kit, was amplified according to manufacturer's recommendations. All incubations were made using the thermal cycler \#1294, Techne Progene Peltier. The purification step was done using the Beckman Coulter's Agencourt RNA Clean purification beads (Beckman Coulter, Fullerton, CA, USA). Amplified cDNA was purified using Zymo research DNA Clean\&Concentrator kit (Zymo Research, Orange, CA, USA).

Nugen Technologies, Inc WT-Ovation FF RNA Amplification system V2 (Nugen V2 FF). RNA (5 ng) extracted from FF NSCLC material was amplified. All incubations were done using the thermal cycler \#1294, Techne Progene Peltier, and purification by Beckman Coulter's Agencourt RNA Clean purification beads.

Nugen Technologies, Inc WT- Ovation FF Pico RNA Amplification system, version 1.0 (Nugen PICO FF). RNA (5 ng) extracted from FF NSCLC material was amplified according to the manufacturer's recommendations. All incubations were done using the thermal cycler \#1294, Techne Progene Peltier and purifications using Beckman Coulter's Agencourt RNA Clean purification beads. The yields and qualities were measured by Nanodrop.

Nugen Technologies, Inc FL-Ovation cDNA Biotin Module $V 2$. Fragmentation, labelling and hybridization of cDNA from Nugen FFPE, Nugen V2 FF and Nugen Pico FF were performed according to the manufacturer's recommendations. Fragmented and labelled cDNA was hybridized onto Affymetrix human U133 plus 2.0 gene chips, followed by washing and scanning.

Affymetrix one cycle amplification system for $F F$ material $(A f f y F F)$. RNA $(1 \mu \mathrm{g})$ from FF NSCLC biopsy was amplified by Affymetrix (Santa Clara, CA, USA) following the manufacturer's recommendations. In brief, RNA and T7-Oligo (dT) primer was mixed and incubated at $70^{\circ} \mathrm{C}$ for $10 \mathrm{~min}$. Firststrand master mix was added followed by incubation for $2 \mathrm{~min}$ at $42^{\circ} \mathrm{C}$. Superscript II was then added and incubated for $1 \mathrm{~h}$ at $42^{\circ} \mathrm{C}$. Second-strand master mix was prepared and added followed by $2 \mathrm{~h}$ incubation at $16^{\circ} \mathrm{C}$. T4 DNA polymerase and EDTA were added separately. Clean-up of cDNA was done followed by biotin labelling and fragmentation. 10X IVT labelling buffer was added. The mix was incubated at $37^{\circ} \mathrm{C}$ for $16 \mathrm{~h}$. cRNA was fragmented using $5 \mathrm{X}$ fragmentation buffer. Labeled and fragmented cRNA was hybridized onto Affymetrix human U133 plus 2.0 gene chips followed by washing and scanning.

Gene expression analysis. The gene expression chip-file was imported into the GeneSpring GX software (Agilent, Redwood City, CA, USA). Data were normalized using the 50th percentile for each chip (per chip normalization). Intensity range, expression values and relative expression for each set of probes were 

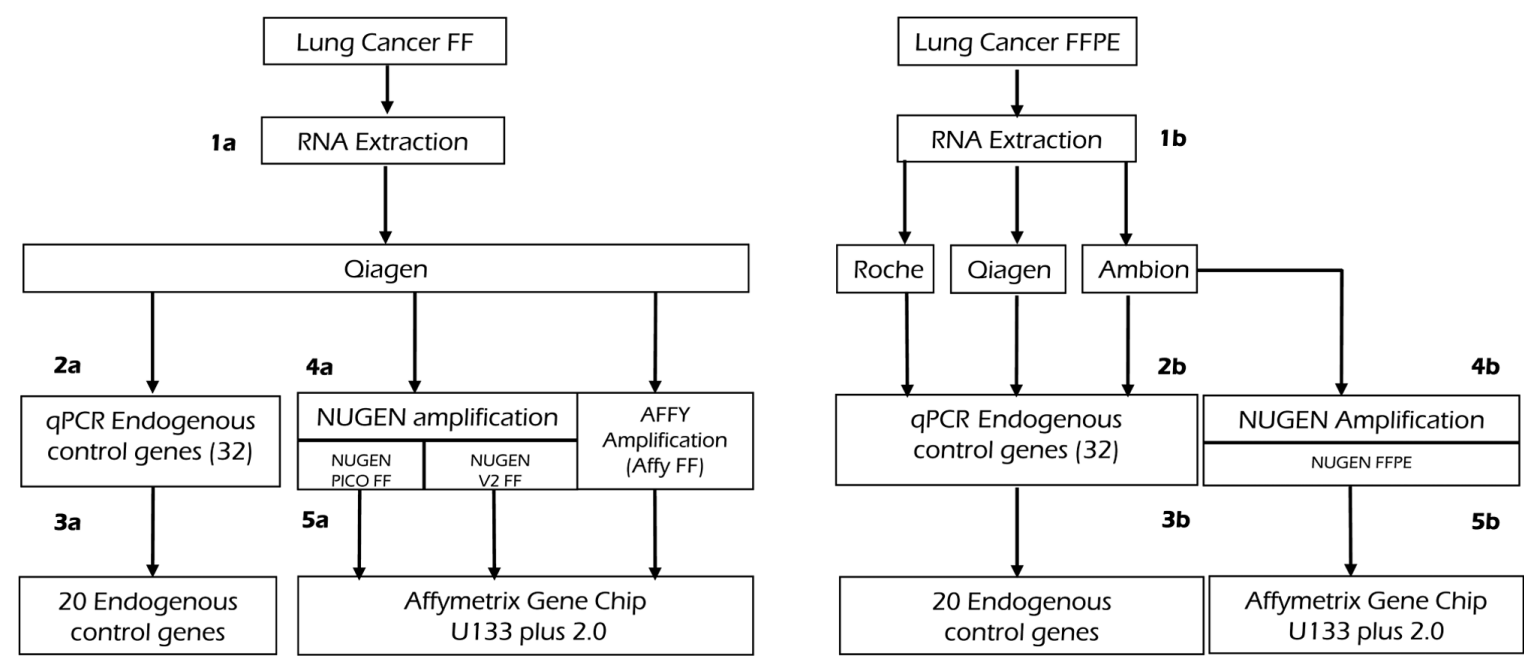

Figure 1. Schematic presentation of the study. The left panel (a) shows the FF procedure and the right panel (b) shows the FFPE procedure. The numbers 1a $\mathrm{and} \mathrm{b}$ to $5 \mathrm{a}$ and $\mathrm{b}$ indicate the order of the individual experiments.

established by normalization over the median of the entire experiment set (per gene normalization). Data filtration based on flags present in at least one of the samples was performed. Gene lists were categorized according to biological functions as described in GeneSpring, NetAffx database (Affymetrix Santa Clara, CA, USA) and GeneCards (www.genecard.org). To determine whether the gene expression of FFPE and FF were consistent with known NSCLC gene expression profiles, two separate published data sets were used $(13,14)$.

Statistical analysis. qPCR data of human endogenous control genes were analyzed using Statistica version 9 (Statsoft Inc. Tulsa, OK, USA). Cycle threshold (CT) was compared between the three extraction methods. Genes with $\mathrm{CT}$ value lower than 36 in one or more of the different methods were further analyzed. Genes with CT above 36 in all samples were excluded. Wilcoxon matched pairs test was performed to compare qPCR CT values.

\section{Results}

RNA extraction. The yield and quality of RNA and cDNA extracted from FFPE and FF NSCLC material were measured using Nanodrop. OD ratio (260/280) showed high quality of RNA and cDNA (Table I).

Evaluation of RNA extraction kit using $q P C R$. The number of endogenous control genes included in the statistical analysis was 20 out of 32 based on a cut-off $>36 \mathrm{CT}$ value. qPCR data with CT values are presented in Table II. All kits were analyzed with two concentrations of input cDNA (20 and $100 \mathrm{ng}$ ). CT values obtained from both concentrations were compared for each kit, to evaluate the required amount of input cDNA. A significant difference in CT values for all kits comparing 20 and $100 \mathrm{ng}$ as input cDNA was found: for Ambion (36.78 \pm 3.34 vs. $33.07 \pm 3.04, \mathrm{p}<0.0001)$, Roche $(36.87 \pm 3.84$ vs. $34.76 \pm 3.46$, $\mathrm{p}<0.005)$ and Qiagen $(37.0 \pm 3.4$ vs. $34.3 \pm 2.9, \mathrm{p}<0.005)$ respectively. The CT values were also compared between the kits, for both 20 and $100 \mathrm{ng}$ of input cDNA. There was no significant difference between the different kits when $20 \mathrm{ng}$ was used.
Table I. Quantity and quality of RNA and cDNA extracted from different kits.

\begin{tabular}{lcccc}
\hline Method & $\begin{array}{c}\text { RNA conc } \\
(\mathrm{ng} / \mu \mathrm{l})\end{array}$ & $\begin{array}{c}\text { OD } \\
260 / 280\end{array}$ & $\begin{array}{c}\text { cDNA conc } \\
(\mathrm{ng} / \mu \mathrm{l})\end{array}$ & $\begin{array}{c}\text { OD } \\
260 / 280\end{array}$ \\
\hline Ambion (FFPE) & 165.8 & 2.1 & 2127 & 1.8 \\
Ambion (FFPE) & 335.5 & 2.0 & 2100 & 1.8 \\
Qiagen (FFPE) & 165.4 & 2.0 & 2201 & 1.8 \\
Qiagen (FFPE) & 187.4 & 2.0 & 2560 & 1.8 \\
Roche (FFPE) & 166.3 & 1.9 & 2119 & 1.8 \\
Roche (FFPE) & 167.1 & 1.9 & 2102 & 1.8 \\
Qiagen (FF) & 419.7 & 2.1 & 2120 & 1.8 \\
Qiagen (FF) & 463.9 & 2.1 & 2326 & 1.8 \\
\hline
\end{tabular}

Numbers show RNA and cDNA concentrations. Ambion, RecoverAll kit; Roche, High Pure FFPE RNA Micro kit; Qiagen, RNeasy FFPE kit; FFPE, formalin-fixed paraffin-embedded.

However, a significant difference in CT values was noted for $100 \mathrm{ng}$ of cDNA comparing Ambion and Roche (33.07 \pm 3.04 vs. 34.76 $\pm 3.46, \mathrm{p}<0.005)$ and Ambion and Qiagen $(33.07 \pm 3.04$ vs. $34.3 \pm 2.9, \mathrm{p}<0.005)$. These results suggest that the Ambion kit, using $100 \mathrm{ng}$ of input material gave the most reliable qPCR results.

Gene expression profiles of FF and FFPE tissue. The global gene expression profile using Affy FF amplification protocol was compared to the Nugen V2 FF, Nugen Pico FF and Nugen FFPE amplification protocols. The variations in normalized data of FFPE Nugen were higher compared to the other amplification methods of FF tissue (Fig. 2). Comparison of Affy FF amplified material with Nugen V2 FF, Nugen Pico FF and Nugen FFPE respectively revealed that Nugen FFPE presented a higher frequency of 2-, 5- and 10-fold up- or downregulated genes compared to Nugen V2 FF and Nugen Pico 
Table II. qPCR data (CT-values) of human endogenous control genes.

\begin{tabular}{lcccccc}
\hline Gene name & $\begin{array}{c}\text { Ambion } \\
\text { FFPE 20 ng }\end{array}$ & $\begin{array}{c}\text { Ambion } \\
\text { FFPE 100 ng }\end{array}$ & $\begin{array}{c}\text { Roche } \\
\text { FFPE 20 ng } \\
\text { Cycle threshold (CT) }\end{array}$ & $\begin{array}{c}\text { Roche } \\
\text { FFPE 100 ng }\end{array}$ & $\begin{array}{c}\text { Qiagen } \\
\text { FFPE 20 ng }\end{array}$ & $\begin{array}{c}\text { Qiagen } \\
\text { FFPE 100 ng }\end{array}$ \\
\hline 18S & 28.05 & 22.66 & 25.22 & 25.86 & 27.18 & 25.32 \\
GAPDH & 36.38 & 34.82 & 35.72 & 35.43 & 40.0 & 37.04 \\
B2M & 32.26 & 30.29 & 32.49 & 31.03 & 32.63 & 30.89 \\
PGK1 & 33.84 & 32.13 & 33.19 & 32.43 & 35.38 & 33.11 \\
RPLP0 & 37.96 & 33.56 & 36.1 & 37.29 & 40.0 & 35.12 \\
TFRC & 40.0 & 34.45 & 40.0 & 40.0 & 40.0 & 36.63 \\
UBC & 33.16 & 31.72 & 35.63 & 33.28 & 34.86 & 32.09 \\
YWHAZ & 40.0 & 36.87 & 40.0 & 40.0 & 40.0 & 34.45 \\
PPIA & 36.13 & 30.22 & 35.91 & 34.18 & 34.56 & 33.6 \\
POLR2A & 40.0 & 35.61 & 40.0 & 32.86 & 35.66 & 35.21 \\
CASC3 & 36.2 & 34.13 & 40.0 & 34.48 & 36.21 & 33.9 \\
CDKN1A & 36.26 & 35.01 & 40.0 & 35.21 & 40.0 & 33.63 \\
CDKN1B & 35.08 & 34.32 & 40.0 & 35.08 & 36.29 & 33.56 \\
EIF2B1 & 40.0 & 35.03 & 40.0 & 36.67 & 34.94 & 35.78 \\
PES1 & 40.0 & 34.05 & 38.26 & 33.49 & 40.0 & 35.96 \\
MT-ATP6 & 35.28 & 31.16 & 33.0 & 31.5 & 35.14 & 34.44 \\
POP4 & 40.0 & 34.46 & 40.0 & 40.0 & 40.0 & 40.0 \\
RPL37A & 40.0 & 34.91 & 35.83 & 36.45 & 40.0 & 36.18 \\
RPL30 & 40.0 & 33.99 & 40.0 & 37.69 & 40.0 & 36.09 \\
RPS17 & 35.07 & 32.08 & 35.81 & 32.31 & 37.07 & 33.57 \\
\hline
\end{tabular}

Numbers show Cycle Threshold values of two different concentrations (20 and 100 ng). Ambion, Recover All kit; Roche, High Pure FFPE RNA Micro kit; Qiagen, RNeasy FFPE kit. FFPE, formalin-fixed paraffin-embedded.

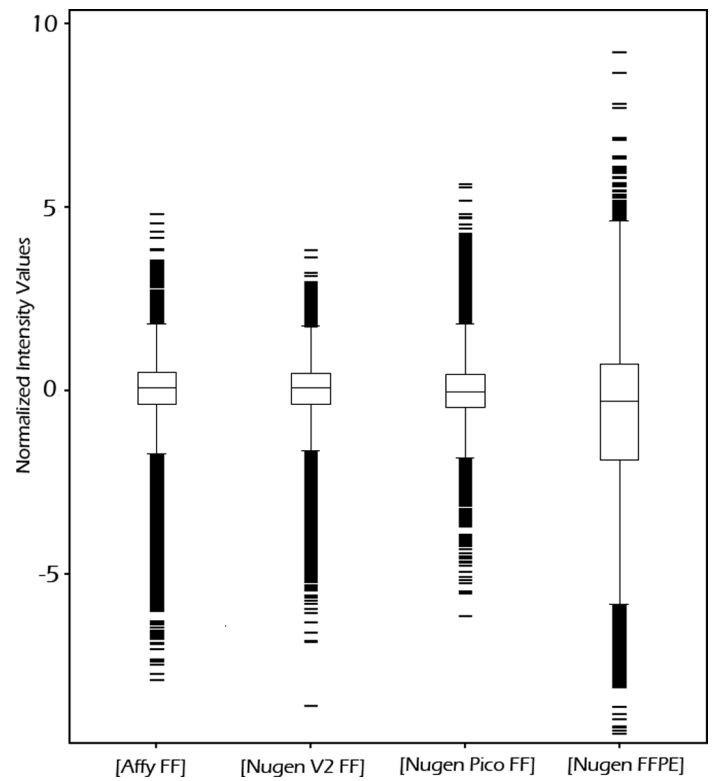

Figure 2. Box plot diagram of the different methods showing normalized intensity values obtained from GeneSpring GX analysis. Nugen FFPE indicates RNA extracted from FFPE and amplified with the Nugen kit. Nugen Pico FF and Nugen V2 FF indicate RNA extracted from FF tissue amplified with Nugen Pico and V2 kits, respectively. Affy FF indicates RNA extracted from $\mathrm{FF}$ tissue amplified with the Affymetrix standard protocol (Affy FF).
FF. Of all genes identified in the Nugen FFPE amplification protocol, $55 \%$ were at least 2-fold up- or down-regulated compared to the Affy FF amplification protocol. Using the Nugen V2 FF and Nugen Pico FF protocols, 23\% and 35\%, respectively of the expressed genes, were at least 2 fold up- or down-regulated compared to the Affy FF amplification protocol.

Sixteen endogenous control genes were selected from the microarray data to compare the amplification methods. A high correlation for the endogenous control genes was noted comparing the Affy FF amplification protocol with Nugen FFPE $(r=0.71, p<0.05)$, Nugen Pico FF $(r=0.88, p<0.05)$ and Nugen V2 FF $(r=0.91, p<0.05)$ amplification protocols respectively (Fig. 3). The microarray data of the 16 endogenous genes were also compared with qPCR data of the same genes. The fold change noted using the Nugen FFPE in qPCR analysis revealed that 14 out of 16 genes were comparable in qPCR and microarray, yielding an $88 \%$ confirmation rate between the data sets (Fig. 4).

Affy FF amplification was compared to Nugen V2 FF, Nugen Pico and Nugen FFPE to analyze the difference in the amplification methods. Previously analyzed FF NSCLC data sets from two publications describing gene signatures specific for NSCLC $(13,14)$ were used. Fifteen common genes in the two publications were selected and compared to our microarray analysis. All 15 genes were identified in our data set comparing 
A

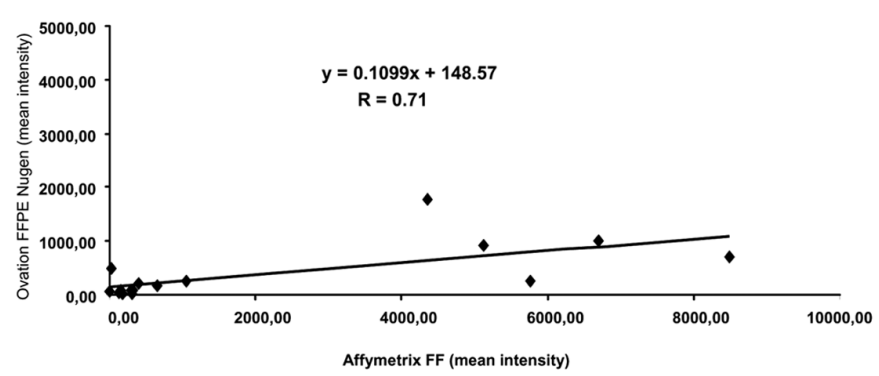

C

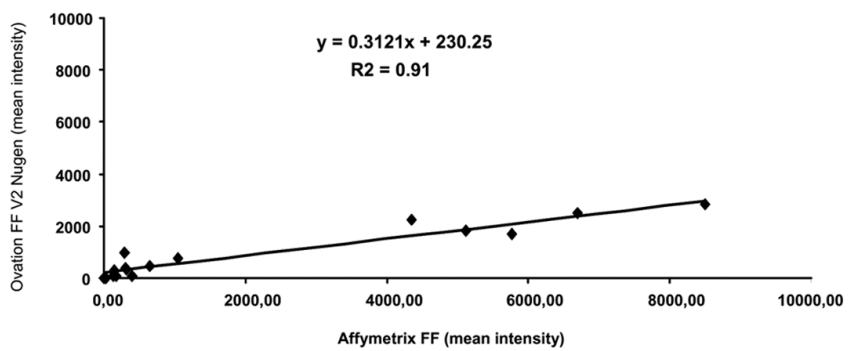

B

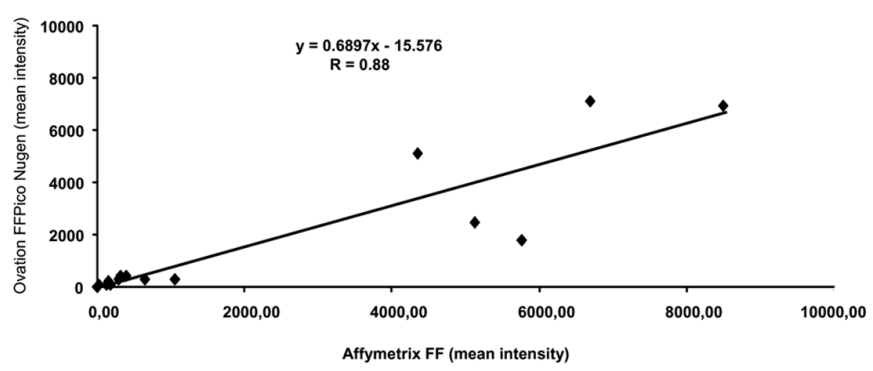

Figure 3. Correlation plot describing FF and FFPE. Correlation between Nugen FFPE (A), Nugen Pico (B) and Nugen FF V2 (C) amplification protocols respectively, compared to the Affy FF standard protocol.

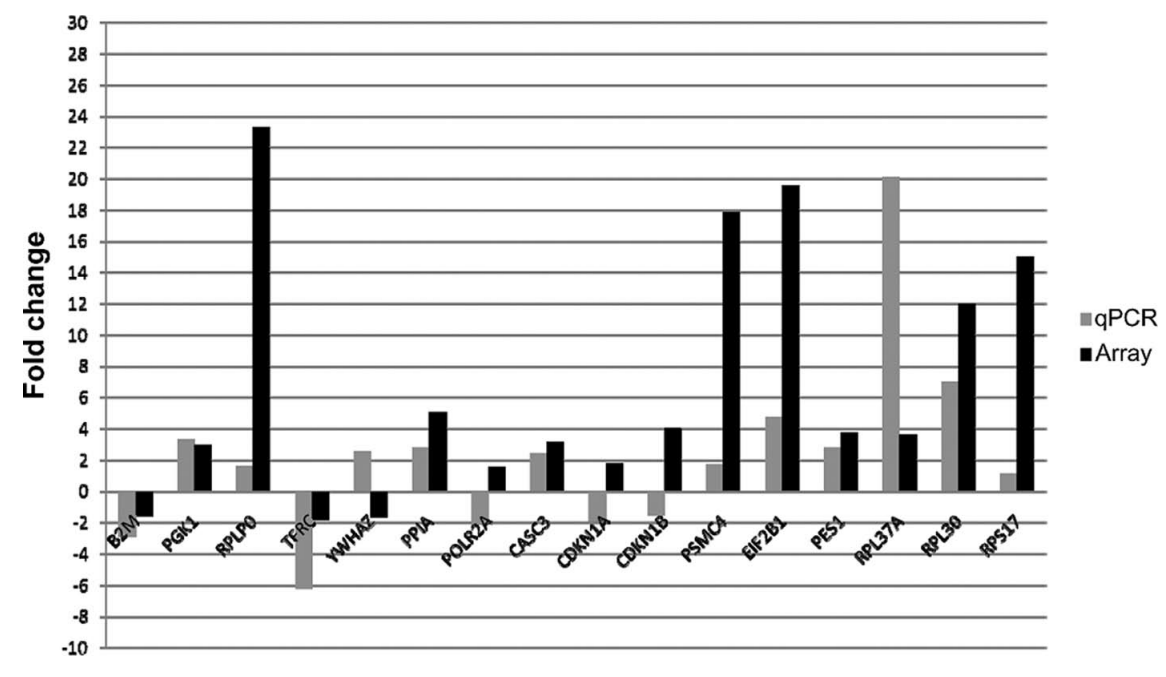

Figure 4. qPCR and microarray fold change data. Comparison of qPCR data (grey box) and microarray (black box) gene expression data of 16 endogenous control genes. Expression less than \pm 2 -fold change indicate no difference between the two groups.

Affy FF to Nugen V2, Nugen Pico and Nugen FFFPE. No difference in gene expression of the 15 selected NSCLC genes was noted between the methods except for one gene (S100P) in Nugen FFPE and one gene (KRT7) in Nugen V2 and Nugen Pico (Fig. 5).

\section{Discussion}

The main aim of the present study was to evaluate methods for long time stored FFPE tissues using gene expression analysis to design an optimal approach for molecular research. We found that the Ambion kit was suitable for RNA extraction of long time stored tissues, due to the lower CT values of qPCR data as compared to Roche and Qiagen. RNA extracted using the Ambion kit was therefore selected for further amplifi- cation using Nugen technologies. Good correlation was found between qPCR and microarray data using endogenous control genes. In addition, known NSCLC gene signatures were identified in both FF and FFPE.

Since FF tissues are limited in number in contrast to stored FFPE tissues in histopathology departments, the evaluation of this material is of importance for molecular research. In the US, there were over 300 million archived cancer tissue samples in 1999, with more samples accumulating at a rate of over 20 million per year (15). However, the use of FFPE RNA has been of limited value in many studies, since the addition of methylol groups by formalin during the process leads to strand breakage and low yield of extracted RNA $(12,16)$.

In the literature, only a few studies have been published that evaluate FFPE RNA extraction methods $(9,17,18)$. Varying 


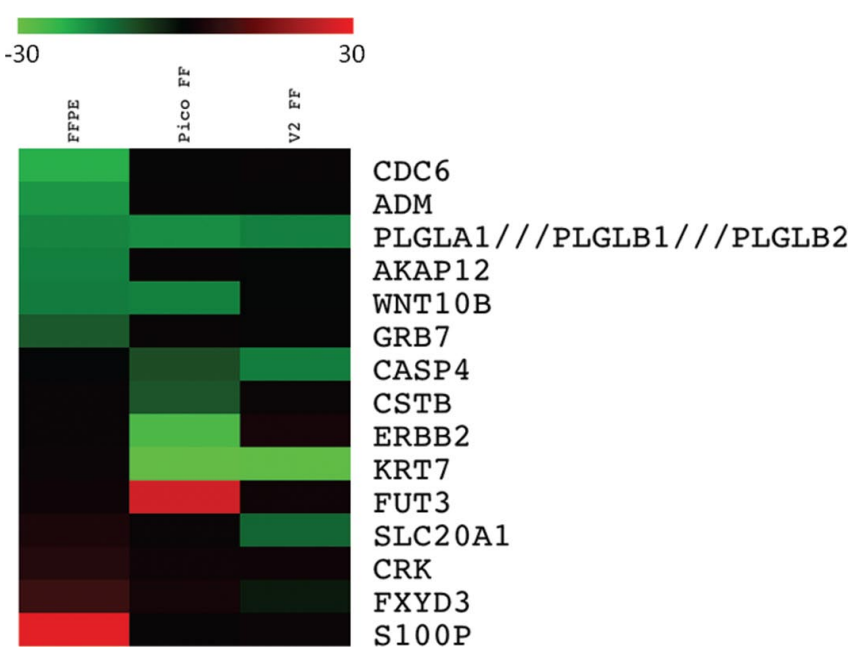

Figure 5. Heat map of NSCLC genes. Comparison of microarray data of 15 NSCLC genes between Nugen FFPE, Nugen Pico and Nugen V2 compared to Affy FF protocol. Red and green, respectively, represents high and low gene expression.

results have been presented as well as criteria of which method that would be most suitable for gene expression analysis. The majority of blocks were stored for less than 8 years and none for more than 15 years. Okello et al (18) used methods from Roche, Ambion, Stratagene and TrimGen and concluded that Ambion was the preferred method, followed by that of Roche. On the other hand Linton et al (17) reported that Qiagen obtained more consistent extraction results compared to Ambion and Fedorowicz et al (9) reported that methods from Ambion and Agencourt, yielded equally high quality of RNA to be used for gene expression. In the present study, RNA extraction of long time stored FFPE was compared using Ambion, Roche and Qiagen, and we report Ambion to be the most suitable kit for gene expression analysis based on qPCR results.

We demonstrate a good concordance comparing the global gene profiles using the Nugen Pico FF, Nugen V2 FF and Nugen FFPE amplification systems. Correlation of expression for the different Nugen amplification kits comparing FFPE and FF material also showed a high degree of specificity, i.e. the expression of genes detected in FFPE material could also be detected in FF material. However, the overall sensitivity of gene expression was lower in FFPE.

Several studies have compared FFPE with FF tissues by gene expression. However, most studies only used qPCR to evaluate the quality of RNA from FFPE using the expression of a small set of selected target genes $(1,7,11,12,16,18,19)$. Specht and colleagues (11) showed that FFPE samples could be used for qPCR gene expression studies with the same reproducibility and precision as frozen samples. In line with this report, Sciccitano et al (10) presented a good correlation between qPCR and microarray using 8 selected genes. They also reported that the majority of gene functions and canonical pathways could be captured from FFPE samples. We report that some genes displayed fold change differences in $\mathrm{qPCR}$ compared to microarray. This is probably due to increased specificity of qPCR analysis compared to microarray and the difference in amplification protocols for qPCR and microarray $(10,20)$. Established NSCLC signatures from two studies $(13,14)$ having 15 genes in common were used as verification of our findings. All 15 genes were identified the present study, both in FF and FFPE specimens and were reported to have no difference in gene expression comparing the amplification protocols used.

The high degree of specificity comparing FFPE with FF materials presented in this study is encouraging and indicates that formalin fixation and paraffin embedding of tissues, can be used for molecular analysis for tissues stored for more than 20 years. Moreover, the methods could be used to isolate high quality RNA from FFPE material for global gene expression analysis using the Affymetrix gene expression platform. Moreover, the use of endogenous control genes seems to be a reliable tool to confirm the quality of gene expression. It is imperative, however, that every step, from RNA extraction to gene expression profiling, is carefully controlled since samples can be degraded throughout the process.

In summary, we have evaluated the performance of long time stored FFPE material using RNA extraction and gene expression. High quality gene expression signatures could be recognized using the Affymetrix gene expression platform on FFPE tissues stored for more than 20 years. The results of this study further confirm the ability to use of FFPE tissues in genomic research and that the storage time of such specimens is not a limiting factor. For samples with storage time above 20 years, the Ambion RecoverAll kit and Nugen amplification system appear to be particularly suited for RNA purification and gene expression analysis, respectively. However, we encourage further studies to evaluate even older tissue specimens to improve the utility for genome-wide RNA-based analysis using FFPE tissues.

\section{Acknowledgements}

We wish to thank L. De Petris and Professor R. Lewensohn (Department of Oncology and Pathology, Karolinska Institutet, Stockholm, Sweden) for contribution of clinical material. This study was supported by grants from Swedish Cancer Foundation, The Cancer Society in Stockholm, King Gustav V Jubilee Fund and the Swedish Research Council. Authors declare no conflict of interest.

\section{References}

1. Abrahamsen HN, Steiniche T, Nexo E, Hamilton-Dutoit SJ and Sorensen BS: Towards quantitative mRNA analysis in paraffinembedded tissuesusingreal-timereverse transcriptase-polymerase chain reaction: a methodological study on lymph nodes from melanoma patients. J Mol Diagn 5: 34-41, 2003.

2. Golub TR: Genome-wide views of cancer. N Engl J Med 344: 601-602, 2001.

3. Benchekroun M, DeGraw J, Gao J, et al: Impact of fixative on recovery of mRNA from paraffin-embedded tissue. Diagn Mol Pathol 13: 116-125, 2004.

4. Masuda N, Ohnishi T, Kawamoto S, Monden M and Okubo K: Analysis of chemical modification of RNA from formalin-fixed samples and optimization of molecular biology applications for such samples. Nucleic Acids Res 27: 4436-4443, 1999.

5. Penland SK, Keku TO, Torrice C, et al: RNA expression analysis of formalin-fixed paraffin-embedded tumors. Lab Invest 87: 383-391, 2007.

6. Srinivasan M, Sedmak D and Jewell S: Effect of fixatives and tissue processing on the content and integrity of nucleic acids. Am J Pathol 161: 1961-1971, 2002. 
7. von Ahlfen S, Missel A, Bendrat $\mathrm{K}$ and Schlumpberger $\mathrm{M}$ : Determinants of RNA quality from FFPE samples. PLoS One 2: E1261, 2007.

8. Bibikova M, Talantov D, Chudin E, et al: Quantitative gene expression profiling in formalin-fixed, paraffin-embedded tissues using universal bead arrays. Am J Pathol 165: 1799-1807, 2004.

9. Fedorowicz G, Guerrero S, Wu TD and Modrusan Z: Microarray analysis of RNA extracted from formalin-fixed, paraffinembedded and matched fresh-frozen ovarian adenocarcinomas. BMC Med Genomics 2: 23, 2009.

10. Scicchitano MS, Dalmas DA, Bertiaux MA, et al: Preliminary comparison of quantity, quality, and microarray performance of RNA extracted from formalin-fixed, paraffin-embedded, and unfixed frozen tissue samples. J Histochem Cytochem 54: 1229-1237, 2006.

11. Specht K, Richter T, Muller U, Walch A, Werner M and Hofler H Quantitative gene expression analysis in microdissected archival formalin-fixed and paraffin-embedded tumor tissue. Am J Pathol 158: 419-429, 2001.

12. von Smolinski D, Leverkoehne I, von Samson-Himmelstjerna G and Gruber AD: Impact of formalin-fixation and paraffinembedding on the ratio between mRNA copy numbers of differently expressed genes. Histochem Cell Biol 124: 177-188, 2005.

13. Beer DG, Kardia SL, Huang CC, et al: Gene-expression profiles predict survival of patients with lung adenocarcinoma. Nat Med 8: 816-824, 2002.
14. Raponi M, Zhang Y, Yu J, et al: Gene expression signatures for predicting prognosis of squamous cell and adenocarcinomas of the lung. Cancer Res 66: 7466-7472, 2006.

15. Bouchie A: Coming soon: a global grid for cancer research. Nat Biotechnol 22: 1071-1073, 2004.

16. Chung JY, Braunschweig T and Hewitt SM: Optimization of recovery of RNA from formalin-fixed, paraffin-embedded tissue. Diagn Mol Pathol 15: 229-236, 2006.

17. Linton K, Hey Y, Dibben S, et al: Methods comparison for highresolution transcriptional analysis of archival material on Affymetrix Plus 2.0 and Exon 1.0 microarrays. BioTechniques 47: 587-596, 2009.

18. Okello JB, Zurek J, Devault AM, et al: Comparison of methods in the recovery of nucleic acids from archival formalin-fixed paraffin-embedded autopsy tissues. Anal Biochem 400: 110-117, 2010.

19. Lehmann U and Kreipe H: Real-time PCR analysis of DNA and RNA extracted from formalin-fixed and paraffin-embedded biopsies. Methods 25: 409-418, 2001.

20. Baum M, Bielau S, Rittner N, et al: Validation of a novel, fully integrated and flexible microarray benchtop facility for gene expression profiling. Nucleic Acids Res 31: E151, 2003. 